\title{
Assessing the Effect of Clinical Inertia on Diabetes Outcomes: a Modeling Approach
}

\author{
Maria F. Correa, $\mathrm{MS}^{7}$, Yan Li, PhD ${ }^{2,3}$, Hye-Chung Kum, PhD ${ }^{4,5,6}$, and Mark A. Lawley, PhD ${ }^{5,6}$ \\ 'Department of Psychiatry, The University of Texas at Austin Dell Medical School, Austin, TX, USA; ${ }^{2}$ Center for Health Innovation, The New York \\ Academy of Medicine, New York, NY, USA; ${ }^{3}$ Department of Population Health Science and Policy, Icahn School of Medicine at Mount Sinai, New \\ York, NY, USA; ${ }^{4}$ Department of Health Policy and Management, Texas A\&M University, College Station, TX, USA; ${ }^{5}$ Center for Remote Health \\ Technologies and Systems, Texas A\&M University, College Station, TX, USA; 'Department of Industrial and Systems Engineering, Texas A\&M \\ University, College Station, TX, USA.
}

BACKGROUND: There are an increasing number of newer and better therapeutic options in the management of diabetes. However, a large proportion of diabetes patients still experience delays in intensification of treatment to achieve appropriate blood glucose targets-a phenomenon called clinical inertia. Despite the high prevalence of clinical inertia, previous research has not examined its long-term effects on diabetes-related health outcomes and mortality.

OBJECTIVE: We sought to examine the impact of clinical inertia on the incidence of diabetes-related complications and death. We also examined how the impact of clinical inertia would vary by the length of treatment delay and population characteristics.

DESIGN: We developed an agent-based model of diabetes and its complications. The model was parameterized and validated by data from health surveys, cohort studies, and trials.

SUBJECTS: We studied a simulated cohort of patients with diabetes in San Antonio, TX.

MAIN MEASURES: We examined 25-year incidences of diabetes-related complications, including retinopathy, neuropathy, nephropathy, and cardiovascular disease.

KEY RESULTS: One-year clinical inertia could increase the cumulative incidences of retinopathy, neuropathy, and nephropathy by $7 \%, 8 \%$, and $18 \%$, respectively. The effects of clinical inertia could be worse for populations who have a longer treatment delay, are aged 65 years or older, or are non-Hispanic whites.

CONCLUSION: Clinical inertia could result in a substantial increase in the incidence of diabetes-related complications and mortality. A validated agent-based model can be used to study the long-term effect of clinical inertia and, thus, inform clinicians and policymakers to design effective interventions.

Electronic supplementary material The online version of this article (https://doi.org/10.1007/s11606-018-4773-3) contains supplementary material, which is available to authorized users.

Received January 15, 2018

Revised August 10, 2018

Accepted November 21, 2018

Published online December 18, 2018
KEY WORDS: diabetes; clinical inertia; diabetes complications; agentbased modeling.

J Gen Intern Med 34(3):372-8

DOI: $10.1007 / \mathrm{s} 11606-018-4773-3$

(C) Society of General Internal Medicine 2018

\section{INTRODUCTION}

Diabetes is a metabolic disease featuring high blood glucose levels over extended periods. In $2010,6.4 \%$ of the global population was affected, with estimates reaching $7.7 \%$ by $2030 .{ }^{1}$ In $2012,9.3 \%$ of the US population (29.1 million) suffered from diabetes, with the expected prevalence growing to $33 \%$ by $2050 .^{2,3}$ In the state of Texas, diabetes is the sixth leading cause of death with approximately $10.6 \%$ of the population impacted directly by the disease. ${ }^{4}$ The prevalence of diabetes is disproportionally higher in urban areas in Texas. For example, more than $14 \%$ of the population in San Antonio has diabetes, and the prevalence is even higher among certain population subgroups (e.g., African Americans and Hispanics). ${ }^{4}$

Previous research has shown that $33 \%$ to $50 \%$ of diabetes cases are undiagnosed or uncontrolled. ${ }^{3}$ This further threatens population health since severe complications such as heart disease, renal failure, and stroke are highly associated with uncontrolled diabetes. ${ }^{5,6}$ Although an increasing number of therapeutic options are becoming available to control diabetes and reduce the risk of severe complications, many patients still experience significant delays in the intensification of treatment-a phenomenon called clinical inertia - which significantly worsens the health outcomes and quality of life for people with diabetes. ${ }^{7}$ For patients who are using insulin, clinical inertia means failure to increase insulin doses or change insulin type to achieve the treatment target. ${ }^{7}$ A recent European study reported an average time to treatment intensification of 3 years among patients with $\mathrm{HbAlc}$ levels greater than $7 \%{ }^{8}$ Another retrospective cohort study found that the mean $\mathrm{HbAlc}$ value at treatment intensification was as high as $9.7 \%$, which was far from the recommended level of less than $7 \% .^{9}$ These significant delays in treatment intensification could cause up to 200,000 avoidable diabetes-related complications per year in the USA. ${ }^{10}$ 
Clinical inertia can result from many factors related to providers, patients, and the health care system. ${ }^{7,}{ }^{11}$ In the provider level, clinical inertia can occur due to a lack of knowledge and training, time constraints, or patient health concerns. For example, patients who are cared for by general practitioners were 2.95 times more likely to experience clinical inertia compared to patients treated by specialists. ${ }^{11}$ The delay in treatment intensification can occur in the patient level due to, for example, concerns about weight gain and hypoglycemia. ${ }^{7}$ Clinical inertia can also be an outcome of system-level factors such as poor access to health care services and expensive new medications. ${ }^{7}$

Although previous studies have revealed the high prevalence of clinical inertia in diabetes treatment and its adverse consequences on short-term patient outcomes, ${ }^{10,11}$ to the best of our knowledge, no previous studies have examined the long-term effects of clinical inertia on diabetes-related complications. Also, there is a lack of knowledge on how the impact of clinical inertia would vary by the length of treatment delay and population characteristics. The primary purpose of this study is to determine the long-term impacts of clinical inertia on diabetesrelated complications across different populations using an agent-based model. We focus on San Antonio, TX, a large city with a diverse population suffering from a high prevalence of diabetes. Specifically, we use an agent-based model to test the impact of clinical inertia on the incidence of diabetes-related complications, including retinopathy, neuropathy, nephropathy, and cardiovascular disease (CVD). We also use the model to test the impact of interactions between clinical inertia and population characteristics (e.g., age, race/ethnicity) on the incidence of diabetes-related complications.

\section{METHODS}

We developed an agent-based model of diabetes progression to project the long-term health impact of different clinical inertia scenarios for people with diabetes in San Antonio, TX. Agentbased modeling is a bottom-up simulation approach that has been increasingly used to study complex problems such as disease prevention and control. ${ }^{12,13}$ Using agent-based modeling, researchers can examine how individuals in a population behave as a function of their characteristics, their environment, and their interactions with each other. ${ }^{14}$ It can provide a powerful simulation lab in which a variety of public health programs, interventions, and policies can be evaluated and compared over a time horizon. When studying chronic diseases, the modeling approach can be used to capture individual health behaviors (e.g., diet, physical activity) and treatment options, as well as other factors (e.g., demographics, neighborhood environment) that may impact the occurrence of the specific condition. ${ }^{12}$ As a result, agent-based modeling can be used to evaluate the longterm impacts of different interventions and draw valuable insights on which intervention is more effective on which population ${ }^{15}$ (for an in-depth discussion of agent-based modeling for chronic disease, see Li et al. ${ }^{12}$ and Nianogo et al. ${ }^{13}$ ).

\section{Model Structure}

We developed an agent-based model of diabetes for this study taking into account our previous work on agent-based modeling of cardiovascular health. ${ }^{16-18}$ In our previous work, we included seven of the most important risk factors for cardiovascular disease - healthy diet, physical activity, smoking status, body mass index (BMI), blood glucose, blood pressure, and cholesterol level - and captured the interconnected relationship between these factors and the incidence of myocardial infarction (MI) and stroke. ${ }^{16}$ In the agent-based model of diabetes, we keep these important risk factors and also include a series of health states representing the development and consequences of diabetes and related complications (i.e., neuropathy, nephropathy, retinopathy, cardiovascular disease).

We use evidence-based equations that guide dynamic changes of individual-level biomarkers (e.g., body mass index, HbA1c) and health factors (e.g., smoking status, hypertension), as well as the impact of these factors on health outcomes. All the equations and model parameters come from critical analysis of existing diabetes simulation models and risk calculators such as the CDC-RTI Diabetes Costeffectiveness Model, the Michigan Model for Diabetes, and the United Kingdom Prospective Diabetes Study (UKPDS) Risk Engine, ${ }^{19-21}$ as well as the best available evidence from the recent literature. The model is implemented by using advanced simulation software-AnyLogic 7. Detailed description of the equations and parameters used in the agentbased model is included in the online supplemental document.

\section{Glycemic Control and Treatment}

Diabetes management is focused on the timely and effective management of glucose levels. Thus, it was important for our agent-based model to capture the continuous changes in HbAlc levels and the process of step-wise intensification of treatment for each individual with diabetes. According to the standards of medical care published by the American Diabetes Association (ADA), diabetes patients should have their HbAlc tested every 3 months, which then determines if the individual requires treatment intensification. Thus, the model uses 3 months as a basic simulation time step. At each time step, the HbA1c value is updated based on the status of treatment intensification and other disease-related characteristics (e.g., age, sex, race/ethnicity, and time since diagnosis).

We considered different medication stages for treatment intensification in the model. Specifically, there are four medication stages based on the standards of medical care recommended by the ADA - monotherapy, dual therapy, triple therapy, and combination injectable therapy - and each person can only receive one type of medication during a treatment intensification event. ${ }^{22}$ If a person's glycemic level is not controlled for receiving one type of medication, he/she will receive a next stage medication for treatment intensification. We present details for glycemic control and treatment intensification in the online supplemental document. 


\section{Model Validation}

To ensure the credibility of simulation results, we conducted systematic model validation based on published model validation guidelines. ${ }^{23,}{ }^{24}$ First, we conducted face and internal validation by examining the accuracy of programming code and consulting with experts in diabetes management. We then conducted external and predictive validation by comparing the simulation results from the agent-based model with those published in clinical trials. Specifically, we selected 14 outcome measures from five published clinical trials for model validation. ${ }^{25-29}$ To simulate the outcomes of each of the clinical trials, we generated a population cohort with similar baseline characteristics, which were provided in each of the trials, and then modeled the development of diabetes-related complications for the same follow-up durations as in the trial.

We plotted simulation results against clinical results for the 14 outcomes in one figure to assess the overall validity of our model (Fig. 1). We conducted linear regression for these outcomes. The slope of the regression line is 0.795 (close to 1), which indicates our model produces similar mean outcomes. The R-squared value is 0.9065 , which indicates that our model explains approximately $91 \%$ of the variance in the clinical data. Thus, the agent-based model presented satisfactory validity in replicating clinical trials. Detailed validation results are included in the online supplemental document.

\section{Simulation Experiments}

We designed simulation experiments to assess the impact of clinical inertia on diabetes-related complications for people with diabetes in San Antonio, TX. We examined how the impact of clinical inertia varies based on different factors, including the duration of clinical inertia, population age, gender, and race/ethnicity. To assess the long-term impact of clinical inertia, we calculated the 25 -year cumulative incidences of neuropathy, retinopathy, nephropathy, MI, stroke, and death due to cardiovascular disease (CVD).

For each simulation experiment, we first created 10,000 simulated individuals with diabetes based on the population characteristics in San Antonio. Specifically, we collected data on age, sex, race, BMI, HbAlc, smoking status, physical activity status, and dietary behaviors from the County Health Rankings and the Texas Demographic Center. ${ }^{30}$ We then simulated diabetes progression for this population for 25 years and counted the incidences of complications. We replicated each simulation experiment 100 times and calculated the $95 \%$ confidence bounds for each outcome to account for uncertainty due to the stochastic nature of the agent-based simulation model.

We projected the incidences of diabetes-related complications for different durations of clinical inertia, including 1, 3, and 7 years. The duration of clinical inertia represented the time that an individual remained in poor glycemic control before the first treatment intensification. We also projected the incidences of diabetes-related complications across different population groups stratified by age, gender, and race/ethnicity under 3year clinical inertia. Three years is an average duration of clinical inertia in the USA based on a large retrospective cohort study. ${ }^{9}$ To assess the extent to which clinical inertia increases the incidence of complications, we simulated a control group with no clinical inertia (no delay in treatment intensification). We performed two-sample $t$ tests on the outcomes of different clinical inertia durations vs. the control group. The two-sample $t$ test is designed to compare the means of two populations while assuming the response variables were normally distributed and the data from both populations have equal variances. In the case of not normally distributed data, we used the Mann-Whitney statistical tests, which use the median to make the comparisons instead of the mean.

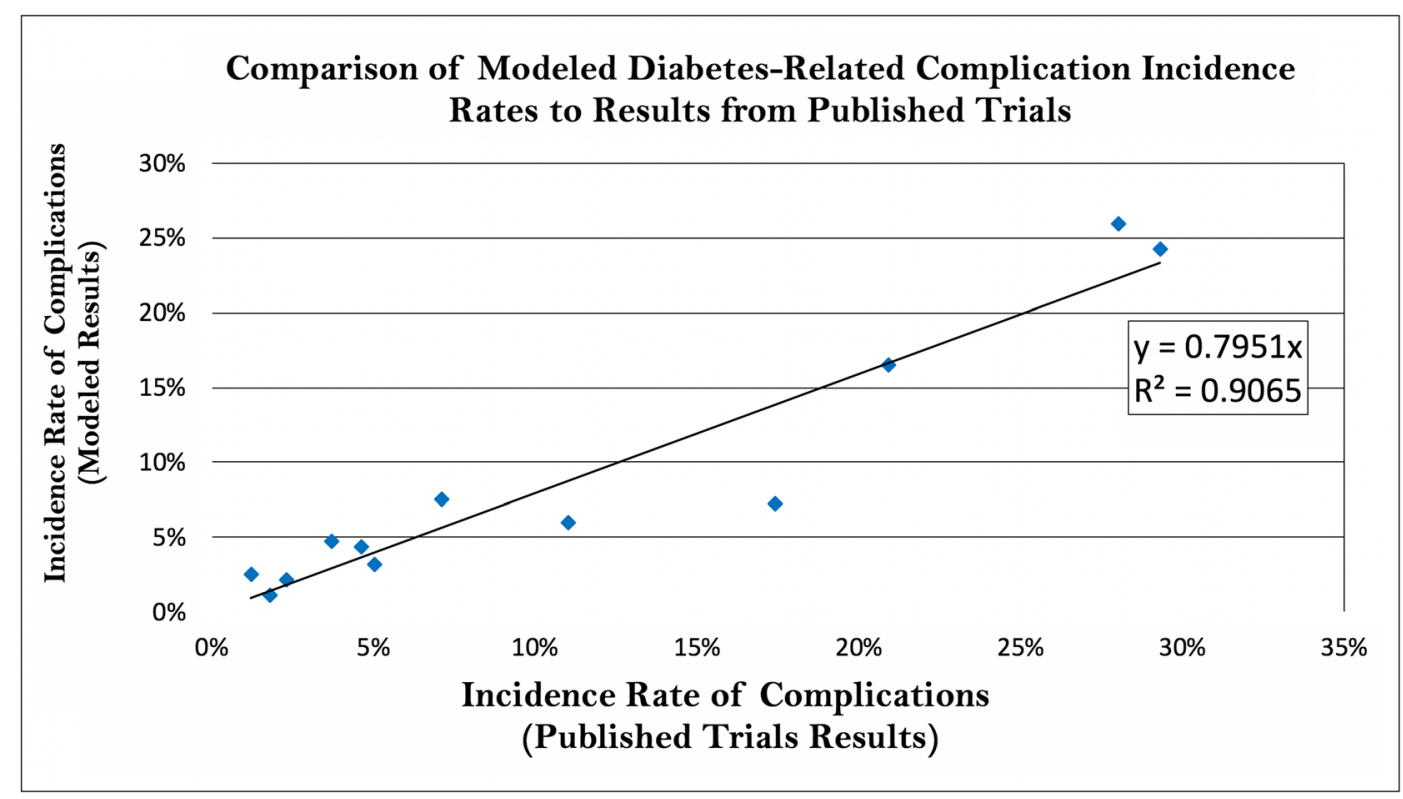

Figure 1 Comparison of the modeled diabetes-related complication incidence rates to the observed results from published trials. 
Table 1 25-Year Cumulative Incidences Under Different Clinical Inertia Scenarios

\begin{tabular}{|c|c|c|c|c|}
\hline & No clinical inertia & $\begin{array}{l}\text { 1-year inertia } \% \\
\text { increase in complication }\end{array}$ & $\begin{array}{l}\text { 3-year inertia \% } \\
\text { increase in complication }\end{array}$ & $\begin{array}{l}\text { 7-year inertia \% } \\
\text { increase in complication }\end{array}$ \\
\hline 25-year cumulative incidences & Mean $(\mathrm{SD}) *$ & Mean $(95 \% \mathrm{CI}) *$ & Mean $(95 \% \mathrm{CI})$ & Mean $(95 \% \mathrm{CI})$ \\
\hline $\mathrm{MI}$ & $435.8(16.1)$ & $8 \%(4 \%, 13 \%)$ & $35 \%(30 \%, 39 \%)$ & $80 \%(76 \%, 84 \%)$ \\
\hline Stroke & $272.6(18.1)$ & $8 \%(3 \%, 15 \%)$ & $30 \%(24 \%, 35 \%)$ & $76 \%(70 \%, 83 \%)$ \\
\hline Neuropathy & $2371(39.5)$ & $8 \%(6 \%, 10 \%)$ & $28 \%(27 \%, 30 \%)$ & $65 \%(64 \%, 67 \%)$ \\
\hline Retinopathy & $874.5(32.4)$ & $7 \%(4 \%, 10 \%)$ & $30(24 \%, 33 \%)^{\ddagger}$ & $71 \%(67 \%, 75 \%)$ \\
\hline Nephropathy & $2185.2(32.9)$ & $16 \%(14 \%, 17 \%)$ & $54 \%(52 \%, 56 \%)$ & $104 \%(103 \%, 106 \%)$ \\
\hline Fatal MI & $13.90(3.60)$ & $2 \%(-34 \%, 30 \%)^{\dagger}$ & $12 \%(-16 \%, 39 \%)^{\dagger}$ & $35 \%(10 \%, 61 \%)$ \\
\hline Death & $1434(23.8)$ & $4 \%(2 \%, 5 \%)$ & $7 \%(6 \%, 8 \%)$ & $14 \%(12 \%, 15 \%)$ \\
\hline Cardiovascular disease death & $247.7(17.8)$ & $15 \%(8 \%, 23 \%)$ & $45 \%(40 \%, 51 \%)$ & $98 \%(91 \%, 104 \%)$ \\
\hline
\end{tabular}

*Mean cumulative incidence (standard deviation) was calculated from 15 replications; incidence increases from the non-clinical inertia group to clinical inertia group (95\% confidence intervals)

$\uparrow P$ value $>0.05$, no statistically significant difference

†Mann-Whitney results for non-normal distribution

\section{RESULTS}

Table 1 reports the incidences of diabetes-related complications for the control group with no clinical inertia and the percent increase in the incidence of complications between the control group and clinical inertia groups for 1, 3, and 7 years. For each simulation run, we used the same characteristics for people with diabetes in San Antonio and only varied the duration of clinical inertia. For the simulated population, the 25year cumulative incidences for MI, stroke, neuropathy, retinopathy, and nephropathy were 436, 273, 2371, 874, and 2185 , respectively. The two-sample $t$ tests showed that there was a statistically significant difference between the control and all clinical inertia groups, in which the clinical inertia scenarios had a higher cumulative incidence for all complications. For example, 1year clinical inertia increased the 25-year cumulative incidence by $8 \%$ (95\% CI 4\%, 13\%), and 3-year and 7 -year clinical inertia increased the incidence rates by $35 \%(95 \%$ CI $30 \%, 39 \%)$ and $80 \%$ (95\% CI $76 \%$, $84 \%$ ), respectively.

\section{Impact of the Interaction Between Clinical Inertia and Age on the Incidence of Complications}

We assessed the impact of clinical inertia on people of three age groups, which include 20-44 years (young adults), 4564 years (midlife adults), and 65 and older (senior adults). We used two-sample $t$ tests to compare the mean incidences between the control and the clinical inertia scenario of each age group. Table 2 shows that the clinical inertia group had significantly higher incidences on all complications than the control group. We also conducted one-way ANOVA tests to compare the mean percent increase from each clinical inertia group to study the different effects of clinical inertia between age groups. We found that the 65 and older age group was affected by clinical inertia significantly more than the 45-64 age group in the incidences of MI, stroke, and neuropathy by $44 \%$ (95\% CI 42\%, 46\%), 44\% (95\% CI 41\%, 46\%), and 47\% (95\% CI 45\%, 49\%), respectively. The 45-64 age group was also affected by clinical inertia significantly more than the 20 44 age group in the incidences of retinopathy, nephropathy, and complication-related deaths by $18 \%(95 \%$ CI 17\%, 20\%),

Table 2 25-Year Cumulative Incidences for Different Age Groups

\begin{tabular}{|c|c|c|c|c|c|c|}
\hline & \multicolumn{2}{|l|}{ Ages 20-44 } & \multicolumn{2}{|l|}{ Ages 45-64 } & \multicolumn{2}{|l|}{ Ages 65-100 } \\
\hline & $\begin{array}{l}\text { No clinical } \\
\text { inertia }\end{array}$ & $\begin{array}{l}\text { 3-year inertia } \\
\% \text { increase in } \\
\text { complications }\end{array}$ & $\begin{array}{l}\text { No clinical } \\
\text { inertia }\end{array}$ & $\begin{array}{l}\text { 3-year inertia } \\
\% \text { increase in } \\
\text { complications }\end{array}$ & $\begin{array}{l}\text { No clinical } \\
\text { inertia }\end{array}$ & $\begin{array}{l}\text { 3-year inertia } \\
\% \text { increase in } \\
\text { complications }\end{array}$ \\
\hline 25-year cumulative incidences & Mean $(\mathrm{SD}) *$ & Mean $(95 \% \mathrm{CI})^{*}$ & Mean (SD) & Mean $(95 \% \mathrm{CI})$ & Mean (SD) & Mean $(95 \% \mathrm{CI})$ \\
\hline MI & $218.4(18.6)$ & $25 \%(19 \%, 30 \%)^{\dagger}$ & $1343.3(43.3)$ & $22 \%(20 \%, 24 \%)$ & $941.6(26)$ & $44 \%(42 \%, 46 \%)$ \\
\hline Stroke & $143.2(15.0)$ & $19 \%(13 \%, 26 \%)$ & $696.9(16.6)$ & $22 \%(20 \%, 25 \%)$ & $991.8(32.8)$ & $44 \%(41 \%, 46 \%)$ \\
\hline Neuropathy & $3721.9(39.3)$ & $18 \%(17 \%, 19 \%)^{\dagger}$ & $3161.1(27.5)$ & $21 \%(20 \%, 22 \%)$ & $1323.5(36)$ & $47 \%(45 \%, 49 \%)$ \\
\hline Retinopathy & $1419.2(29.1)$ & $19 \%(17 \%, 21 \%)$ & $1321.7(26.6)$ & $18 \%(17 \%, 20 \%)$ & $500.7(16.3)$ & $46 \%(44 \%, 49 \%)^{\dagger}$ \\
\hline Nephropathy & $3430(47.8)$ & $31 \%(30 \%, 32 \%)$ & $2928.5(37)$ & $37 \%(36 \%, 38 \%)$ & $1285.0(36.7)$ & $77 \%(74 \%, 80 \%)^{\dagger}$ \\
\hline Fatal MI & $1.4(1.2)$ & $75 \%(50 \%, 125 \%)^{\dagger}$ & $46.9(8.9)$ & $49 \%(36 \%, 63 \%)$ & $79.4(7.6)$ & $46 \%(37 \%, 54 \%)^{\dagger}$ \\
\hline Complication-related death & $398.2(17.5)$ & $49 \%(45 \%, 52 \%)$ & $1382.7(45.5)$ & $32 \%(29 \%, 35 \%)$ & $1798.1(30.9)$ & $46 \%(45 \%, 47 \%)^{\dagger}$ \\
\hline Cardiovascular disease death & $118.1(10.1)$ & $32 \%(25 \%, 39 \%)$ & $1148.5(39)$ & $28 \%(25 \%, 31 \%)$ & $1738.9(26.4)$ & $45 \%(44 \%, 46 \%)$ \\
\hline
\end{tabular}

*Mean cumulative incidence (standard deviation) was calculated from 15 replications; incidence increases from the non-clinical inertia group to clinical inertia group (95\% confidence intervals)

†Mann-Whitney results for non-normal distribution 
$31 \%(95 \%$ CI $30 \%, 32 \%)$, and $32 \%$ (95\% CI 29\%, 35\%), respectively.

\section{Impact of the Interaction Between Clinical Inertia and Ethnicity/Race on the Incidence of Complications}

We assessed the impact of clinical inertia on people of different races/ethnicities (i.e., non-Hispanic White, African American, Hispanic, Native American, and Asian). We present the simulation results and the two-sample $t$ test results in Table 3 . We found that the incidences of neuropathy and retinopathy for non-Hispanic Whites would increase by $21 \%$ (95\% CI $19 \%, 22 \%)$ and $19 \%(95 \%$ CI 16\%, 22\%), respectively, if they experience clinical inertia. Clinical inertia would increase the incidences of MI and stroke by $25 \%$ (95\% CI 22\%, 28\%) and $24 \%$ (95\% CI 20\%, 29\%), respectively, for the Hispanic population.

\section{Impact of the Interaction Between Clinical Inertia and Gender on the Incidence of Complications}

Finally, we assessed the impact of clinical inertia on people of different genders. The results show that clinical inertia resulted in higher incidence rates for all complications for both males and females. Further tests showed that clinical inertia would affect females more profoundly compared to males. For example, the 25-year cumulative incidences of stroke for females and males were 384 and 331, respectively. Also, 3-year clinical inertia could increase the incidences of stroke by $28 \%$ (95\% CI 23\%, 33\%) for females and 24\% (95\% CI 20\%, 28\%) for males (results are not included in the tables).

\section{DISCUSSION}

Clinical inertia is a critical barrier to the effective treatment of diabetes mellitus. Despite its prevalence, there have been few studies on its effects. A clear understanding of the impact of clinical inertia on diabetes-related complications is critical. Computer modeling and simulation has been increasingly recognized as a practical approach to study different chronic diseases such as diabetes. ${ }^{31}$ This study, for the first time, uses a flexible, agentbased model to assess the long-term impact of clinical inertia.

We found that clinical inertia could increase the incidence of diabetes-related complications significantly among people with diabetes in San Antonio, TX. Our simulation results were broadly consistent with those of the existing clinical studies, which examined the short-term impact of clinical inertia on diabetes-related complications. ${ }^{11,32} \mathrm{We}$ also found that clinical inertia could have different impacts on people of different age groups and races/ethnicities. In particular, people who are

Table 3 25-Year Cumulative Incidences for Different Racial/Ethnic Groups

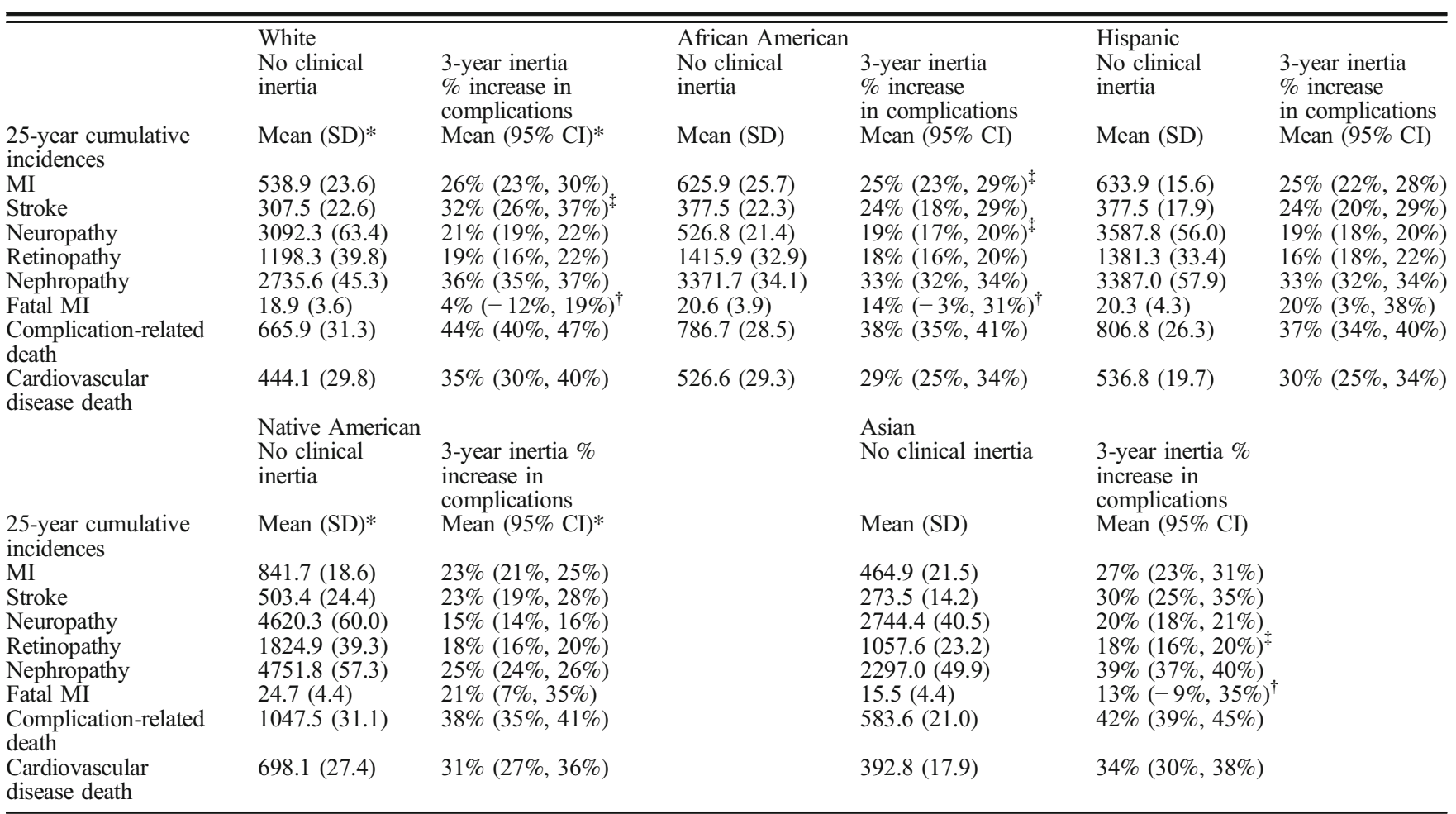

* Mean cumulative incidence (standard deviation) was calculated from 15 replications; incidence increases from the non-clinical inertia group to clinical inertia group (95\% confidence intervals)

$\uparrow P$ value $>0.05$, no statistically significant difference

$\$$ Mann-Whitney results for non-normal distribution 
65 years or older were more vulnerable to clinical inertia compared to their younger counterparts. This is likely because diabetes and its complications affect older adults more than young and midlife adults. Further, interventions that reduce clinical inertia have the potential to reduce racial/ethnic and gender disparities in diabetes outcomes. With the validated agent-based model, we were able to quantify the impact of clinical inertia and, thus, provide actionable evidence on diabetes control and management practices.

Our study has several limitations. First, our model only predicts the first event of diabetes complications, similar to the CDC-RTI model. ${ }^{20}$ Consequently, we are only able to track the cumulative incidence of the first event of diabetes complications. Second, we assume that the treatment intensification decision is only based on the glycemic level of an individual, whereas in reality treatment intensification may be based on other risk factors such as age and overall health. Third, we do not consider potential discontinuation of medication due to a lack of clinical data on discontinuation. In other words, we assume that patients are adhering to their medication instructions. Fourth, we assume that clinical inertia only occurs once, which means once a patient receives treatment intensification, he/she will receive proper treatment based on the ADA guidelines. We will relax this assumption once we have clinical data on patterns of clinical inertia for patients with diabetes. Finally, we did not use the duration of diabetes as a variable in the model, which is consistent with other diabetes simulation models. ${ }^{20}$

Despite these limitations, our agent-based model of diabetes, validated by results from multiple large clinical trials, has the potential to evaluate the impact of different prevention and treatment interventions in the future. In addition, we can adapt the model to simulate the impact of clinical inertia on populations from other places. As a result, targeted interventions - such as incentivizing patients for timely diabetes treatment, promoting telehealth technology for improved communication between patients and physicians, and physician education programs - can be assessed before implementation. Clinical inertia could have different impacts on diabetes-related complications across different population groups. Our modeling approach will help tailor interventions to specific populations, which is a necessary step to reduce health disparities and improve population health.

Corresponding Author: Yan Li, PhD; Center for Health Innovation The New York Academy of Medicine, New York, NY, USA (e-mail: yli@nyam.org).

Funding Information Dr. Li's role in the research reported in this publication was supported, in part, by the National Heart, Lung, And Blood Institute of the National Institutes of Health under Award Number R01HL141427. The content is solely the responsibility of the authors and does not necessarily represent the official views of the National Institutes of Health.

\section{Compliance with Ethical Standards:}

Conflict of Interest: The authors declare that they do not have a conflict of interest.

Publisher's note Springer Nature remains neutral with regard to jurisdictional claims in published maps and institutional affiliations.

\section{REFERENCES}

1. Shaw JE, Sicree RA, Zimmet PZ. Global estimates of the prevalence of diabetes for 2010 and 2030. Diabetes Res Clin Pract 2010;87(1):4-14.

2. Boyle JP, Thompson TJ, Gregg EW, Barker LE, Williamson DF. Projection of the year 2050 burden of diabetes in the US adult population: dynamic modeling of incidence, mortality, and prediabetes prevalence. Popul Health Metrics 2010;8(1):29.

3. Cowie CC, Rust KF, Byrd-Holt DD, et al. Prevalence of diabetes and impaired fasting glucose in adults in the US population National Health and Nutrition Examination Survey 1999-2002. Diabetes Care 2006;29(6):1263-1268.

4. Centers for Disease Control and Prevention. Diabetes Data \& Statistics. Atlanta, GA; 2018. https://www.cdc.gov/diabetes/data/index.html. Accessed August 31, 2018.

5. Li R, Bilik D, Brown MB, et al. Medical costs associated with type 2 diabetes complications and comorbidities. Am J Manag Care 2013;19(5):421.

6. Nathan DM, DCCT Research Group. The diabetes control and complications trial/epidemiology of diabetes interventions and complications study at 30 years: overview. Diabetes Care 2014;37(1):9-16.

7. Lovshin JA, Zinman B. Diabetes: clinical inertia-a barrier to effective management of T2DM. Nat Rev Endocrinol 2013;9(11):635-636.

8. Stone MA, Charpentier G, Doggen $\mathbf{K}$, et al. Quality of care of people with type 2 diabetes in eight European countries. Diabetes Care 2013;36(9):2628-2638.

9. Khunti K, Wolden ML, Thorsted BL, Andersen M, Davies MJ. Clinical inertia in people with type 2 diabetes. Diabetes Care 2013;36(11):34113417.

10. Strain WD, Blüher M, Paldánius $\mathbf{P}$. Clinical inertia in individualising care for diabetes: is there time to do more in type 2 diabetes?, Diabetes Ther 2014;5(2):347-354.

11. Osataphan $\mathbf{S}$, Chalermchai $\mathbf{T}$, Ngaosuwan $\mathbf{K}$. Clinical inertia causing new or progression of diabetic retinopathy in type 2 diabetes: A retrospective cohort study. J Diabetes 2017;9(3):267-274.

12. Li Y, Lawley MA, Siscovick DS, Zhang D, Pagán JA. Agent-Based Modeling of Chronic Diseases: A Narrative Review and Future Research Directions. Prev Chronic Dis 2016;13:150561.

13. Nianogo RA Arah OA. Agent-Based Modeling of Noncommunicable Diseases: A Systematic Review. Am J Public Health 2015;105(3):e20-e31.

14. Epstein JM. Generative Social Science: Studies in Agent-Based Computational Modeling. Princeton University Press, Princeton, NJ; 2006.

15. Rigotti NA, Wallace RB. Using Agent-Based Models to Address "Wicked Problems" Like Tobacco Use: A Report from the Institute of Medicine. Ann Intern Med 2015;163(6):469-471.

16. Li Y, Kong N, Lawley M, Weiss L, Pagán JA. Advancing the Use of EvidenceBased Decision-Making in Local Health Departments With Systems Science Methodologies. Am J Public Health 2015; 105(S2):S217-S222.

17. Li Y, Kong N, Lawley MA, Pagán JA. Using Systems Science for Population Health Management in Primary Care. J Prim Care Community Health 2014;5(4):242-246.

18. Li Y, Kong N, Lawley M, Pagán JA. Assessing lifestyle interventions to improve cardiovascular health using an agent-based model. In: Proceedings of the 2014 Winter Simulation Conference. IEEE Press; 2014:1221-1232.

19. Hoerger TJ, Bethke AD, Richter A, et al. Cost-effectiveness of intensive glycemic control, intensified hypertension control, and serum cholesterol level reduction for type 2 diabetes. JAMA 2002;287(19):2542-2551. 
20. Zhou H, Isaman DJ, Messinger S, et al. A computer simulation model of diabetes progression, quality of life, and cost. Diabetes Care 2005;28(12):2856-2863.

21. Stevens RJ, Coleman RL, Adler AI, Stratton IM, Matthews DR, Holman RR. Risk factors for myocardial infarction case fatality and stroke case fatality in type 2 diabetes UKPDS 66. Diabetes Care 2004;27(1):201-207.

22. American Diabetes Association. Standards of medical care in diabetes-2016 abridged for primary care providers. Clin Diabetes 2016;34(1):3-21.

23. Fagiolo G, Moneta A, Windrum P. A critical guide to empirical validation of agent-based models in economics: Methodologies, procedures, and open problems. Comput Econ 2007;30(3):195-226.

24. Eddy DM, Hollingworth W, Caro JJ, Tsevat J, McDonald KM, Wong JB. Model transparency and validation a report of the ISPOR-SMDM Modeling Good Research Practices Task Force-7. Med Decis Mak 2012;32(5):733-743.

25. Adler AI, Stevens RJ, Manley SE, et al. Development and progression of nephropathy in type 2 diabetes: the United Kingdom Prospective Diabetes Study (UKPDS 64). Kidney Int 2003;63(1):225-232.

26. The Action to Control Cardiovascular Risk in Diabetes Study Group. Effects of intensive glucose lowering in type 2 diabetes. $N$ Engl $J$ Med 2008;2008(358):2545-2559.
27. UK Prospective Diabetes Study (UKPDS) Group. Intensive blood-glucose control with sulphonylureas or insulin compared with conventional treatment and risk of complications in patients with type 2 diabetes (UKPDS 33). Lancet 1998;352(9131):837-853.

28. Li G, Zhang $\mathbf{P}$, Wang $\mathbf{J}$, et al. The long-term effect of lifestyle interventions to prevent diabetes in the China Da Qing Diabetes Prevention Study: a 20-year follow-up study. Lancet 2008;371(9626): 1783-1789.

29. Partanen $\mathbf{J}$, Niskanen $\mathbf{L}$, Lehtinen $\mathbf{J}$, Mervaala $\mathbf{E}$, Siitonen $\mathbf{O}$, Uusitupa M. Natural history of peripheral neuropathy in patients with non-insulin-dependent diabetes mellitus. N Engl J Med 1995;333(2):8994.

30. Robert Wood Johnson Foundation, University of Wisconsin Population Health Institute. County Health Rankings \& Roadmaps. http://www. countyhealthrankings.org. Accessed August 31, 2018.

31. Hoerger TJ, Hicks KA, Sorensen SW, et al. Cost-effectiveness of screening for pre-diabetes among overweight and obese US adults. Diabetes Care 2007;30(11):2874-2879.

32. Paul SK, Klein K, Thorsted BL, Wolden ML, Khunti K. Delay in treatment intensification increases the risks of cardiovascular events in patients with type 2 diabetes. Cardiovasc Diabetol 2015;14(1):100. 